OPEN ACCESS

Edited by:

Michael Walch,

Université de Fribourg,

Switzerland

Reviewed by:

Landon Edgar,

University of Toronto, Canada

Jeremy Alastair O'Sullivan,

Northwestern University,

United States

*Correspondence:

Chitra Mandal

chitra_mandal@yahoo.com

${ }^{\text {t}}$ These authors have contributed equally to this work

Specialty section: This article was submitted to

Molecular Innate Immunity,

a section of the journal

Frontiers in Immunology

Received: 14 August 2021 Accepted: 18 October 2021 Published: 03 November 2021

Citation:

Karmakar J, Mukherjee K and Mandal C (2021) Siglecs

Modulate Activities of Immune Cells Through Positive and Negative Regulation of ROS Generation.

Front. Immunol. 12:758588. doi: 10.3389/fimmu.2021.758588

\section{Siglecs Modulate Activities of Immune Cells Through Positive and Negative Regulation of ROS Generation}

\author{
Joyshree Karmakar ${ }^{\dagger}$, Kaustuv Mukherjee ${ }^{\dagger}$ and Chitra Mandal ${ }^{*}$ \\ Cancer Biology and Inflammatory Disorder Division, Council of Scientific and Industrial Research (CSIR)-Indian Institute of \\ Chemical Biology, Kolkata, India
}

Reactive oxygen species (ROS) are a group of oxygen-containing highly-reactive molecules produced from oxidative metabolic processes or in response to intracellular signals like cytokines and external stimuli like pathogen attack. They regulate a range of physiological processes and are involved in innate immune responses against infectious agents. Deregulation of ROS contributes to a plethora of disease conditions. Sialic acids are carbohydrates, present on cell surfaces or soluble proteins. Sialic acid-binding immunoglobulin-like lectins (Siglecs) recognize and bind to sialic acids. These are widely expressed on various types of immune cells. Siglecs modulate immune activation and can promote or inhibit ROS generation under different contexts. Siglecs promote ROS-dependent cell death in neutrophils and eosinophils while limiting oxidative stress associated with chronic obstructive pulmonary disease (COPD), sickle cell disease (SCD), coronavirus disease-2019 (COVID-19), etc. This review distinguishes itself in summarizing the current understanding of the role of Siglecs in moderating ROS production and their distinct effect on different immune cells; that ultimately determine the cellular response and the disease outcome. This is an important field of investigation having scope for both expansion and medical importance.

\footnotetext{
Keywords: chronic obstructive pulmonary disease, coronavirus disease (COVID-19), Leishmania donovani, neutrophil, reactive oxygen species, siglecs, Siglec-sialoglycan, eosinophil
}

\section{INTRODUCTION}

Sialic acids (SA), a family of nine-carbon acidic monosaccharides, are commonly found at terminal positions in glycan chains attached to glycoproteins/glycolipids (sialoglycans) present on cell surfaces or soluble proteins. So far, nearly 50 different derivatives of SA have been discovered (1). Sialic acids are commonly found in higher mammals but also in several protozoans, bacteria, and fungi (1). Sialic acid-binding immunoglobulin-like lectins (Siglecs) are a class of glycan-binding proteins that recognize and bind to these SA (2-4). Several Siglecs modulate cellular activity and response via signaling through cytoplasmic regulatory motifs.

Reactive oxygen species (ROS) refers to oxygen-derived, highly reactive molecules which include superoxide anion and hydrogen peroxide (5). ROS are produced in mitochondria as by-products of the electron transfer chain during aerobic respiration. Phagocytic cells (macrophages, neutrophils, and 
dendritic cells) also generate ROS and reactive nitrogen species (RNS) as components of "oxidative burst" for degrading biomolecules and internalized pathogens (6).

In this review, we have attempted to highlight how Siglecsialoglycan interactions modulate ROS generation in various immune cells depending on physiological states or infections.

\section{Distribution and Classification of Siglecs}

Most immune cells of hematopoietic origin express one or more kinds of Siglecs (7). Although resting $\mathrm{T}$ cells show low Siglec expression, a few T cell subsets express Siglecs (particularly Siglec-5, 7,9 , and 10) after activation or in specific contexts (8-10) (Table 1). Some Siglecs, like Siglec-9, are expressed on several immune cells. However, expression of certain Siglecs is restricted to particular cell types; like Siglec-1 (Sialoadhesin) on monocytes, macrophages, and dendritic cells (15). Siglec-2 (CD22) is predominantly observed on $\mathrm{B}$ cells though it is also expressed at low levels on mast cells, dendritic cells, and basophils (17-20) (Table 1).

Siglecs are classified into two major groups based on sequence homology. The first group (Siglec-1, CD22, Siglec-4, and

TABLE 1 | Distribution of human and mice siglec families on different cells, ligand preferences and their common functions.

\begin{tabular}{|c|c|c|c|c|}
\hline Siglecs & Distribution on cells & $\begin{array}{l}\text { Linkage preference on } \\
\text { sialylated ligands }\end{array}$ & Functions & References \\
\hline \multicolumn{5}{|c|}{ Evolutionary conserved siglecs } \\
\hline $\begin{array}{l}\text { Siglec-1 } \\
(\mathrm{CD}-169)\end{array}$ & Macrophages & $\alpha 2,3$ & $\begin{array}{l}\text { Recognition and phagocytosis of sialylated pathogens. } \\
\text { Modulates immune response through cell-cell interactions }\end{array}$ & $(7,11-15)$ \\
\hline $\begin{array}{l}\text { Siglec-2 } \\
\text { (CD22) }\end{array}$ & $\begin{array}{l}\text { Predominantly on B-cells, also } \\
\text { detected in } \\
\text { Dendritic cells } \\
\text { Mast cell } \\
\text { Basophils, } \\
\text { Gut eosinophils of mouse }\end{array}$ & $\begin{array}{l}\alpha 2,6 \text { - preferably, Neu5Gc/ } \\
\text { Neu5Ac }\end{array}$ & Regulates B cell survival, signaling and homeostasis & $\begin{array}{l}(7,11,12 \\
14,16-20)\end{array}$ \\
\hline $\begin{array}{l}\text { Siglec-4 } \\
\text { (MAG) }\end{array}$ & Neuronal cells & $\alpha 2,3>\alpha 2,6$ & $\begin{array}{l}\text { Secures myelin-axon associations through binding with } \\
\text { axonic gangliosides GD1a and GT1b. } \\
\text { Regulates axon growth and survival }\end{array}$ & $(7,12)$ \\
\hline Siglec-15 & Osteoclasts, macrophages & $\alpha 2,6$ & $\begin{array}{l}\text { Regulates osteoclast differentiation, involved in regulation of } \\
\text { immune response }\end{array}$ & $(11-13,16)$ \\
\hline \multicolumn{5}{|c|}{ Siglec-3 (CD-33) related siglecs } \\
\hline Siglec-3 & Myeloid progenitors & $\alpha 2,6>\alpha 2,3$ & Involved in immune inhibitory functions & $(7,11,12)$ \\
\hline Siglec-5 & $\begin{array}{l}\text { Monocytes, neutrophils, } \\
\text { activated T cells }\end{array}$ & $\alpha 2,3$ & $\begin{array}{l}\text { Involved in pathogen phagocytosis and clearance of sialylated } \\
\text { substrates }\end{array}$ & $(7,9,10,12)$ \\
\hline Siglec-6 & $\begin{array}{l}\text { Trophoblasts, } \\
\text { Mast cells } \\
\text { B cells }\end{array}$ & $\alpha 2,6$ - is preferred & $\begin{array}{l}\text { Highest expression levels in placenta but function in gestation } \\
\text { still not explored }\end{array}$ & $(11,12)$ \\
\hline Siglec-7 & $\begin{array}{l}\text { Neutrophils, } \\
\text { monocytes, } \\
\text { mast cells, NK cells, CD8-T cell } \\
\text { subset }\end{array}$ & $\alpha 2,8-$ is preferred & Inhibitory in nature, down regulates T cell signaling & $(11,12)$ \\
\hline $\begin{array}{l}\text { Siglec-8 } \\
\text { (Human) }\end{array}$ & $\begin{array}{l}\text { Eosinophils, basophils, } \\
\text { mast cells }\end{array}$ & $\begin{array}{l}\alpha 2,3-\text { and sulphated } \\
\text { ligands }\end{array}$ & Involved in cellular apoptosis & $(7,12)$ \\
\hline Siglec- $\mathrm{F}$ & Eosinophils & & & \\
\hline $\begin{array}{l}\text { (closely related functional } \\
\text { murine paralog) }\end{array}$ & Macrophages & & & \\
\hline $\begin{array}{l}\text { Siglec-9 } \\
\text { (Human) }\end{array}$ & $\begin{array}{l}\text { Neutrophils, monocytes, } \\
\text { NK cells, dendritic cells, B cells, } \\
\text { CD8-T cell subset }\end{array}$ & $\begin{array}{l}\alpha 2,3 \text { or } \alpha 2,6-\text { or sulfated } \\
\text { ligands }\end{array}$ & $\begin{array}{l}\text { Involved in cellular response apoptosis and inhibition of } \\
\text { immune response }\end{array}$ & $(7,9,10,12)$ \\
\hline $\begin{array}{l}\text { Siglec-E } \\
\text { (murine paralog) }\end{array}$ & $\begin{array}{l}\text { Neutrophils } \\
\text { Monocytes } \\
\text { dendritic cells }\end{array}$ & & & \\
\hline $\begin{array}{l}\text { Siglec-10 } \\
\text { (Human) }\end{array}$ & $\begin{array}{l}\text { NK cells, B cells, } \\
\text { monocytes } \\
\text { eosinophils, T cells }\end{array}$ & $\alpha 2,3$ or $\alpha 2,6$ & Inhibits calcium signalling mediated by B cell receptor & $(7,10,11)$ \\
\hline $\begin{array}{l}\text { Siglec-G } \\
\text { (Murine paralog) }\end{array}$ & $\begin{array}{l}\text { B cells, } \\
\text { dendritic cells }\end{array}$ & & & \\
\hline Siglec-11 & $\begin{array}{l}\text { Macrophage, B cells, microglia, } \\
\text { ovary stroma }\end{array}$ & $\alpha 2,8-$ is preferred & Involved in pathology of neurodegenerative diseases & $(7,11)$ \\
\hline $\begin{array}{l}\text { Siglec-14 } \\
\text { (Human) }\end{array}$ & Monocytes, neutrophils & $\alpha 2,3$ & $\begin{array}{l}\text { Functions as an activating receptor through association with } \\
\text { DNAX Activating Protein of } 12 \mathrm{kDa} \text { (DAP12) }\end{array}$ & $(7,14)$ \\
\hline $\begin{array}{l}\text { Siglec-H } \\
\text { (Murine paralog) }\end{array}$ & $\begin{array}{l}\text { Monocytes } \\
\text { neutrophils Microglia }\end{array}$ & & & \\
\hline Siglec-16 & Microglia & $\alpha 2,8-$ is preferred & Involved in Pathology of neurodegenerative disease & $(7,11)$ \\
\hline
\end{tabular}

This table has been compiled from information collected from references \# (7, 9-20). The distribution of siglecs on human and murine immune cells is indicated here. 
Siglec-15) has low sequence similarity between each other but is conserved across species. In contrast, CD33-related Siglecs (CD33r Siglecs) are closely related but not highly conserved (21). Siglecs have been extensively studied in humans, mice, and few other animals. Humans express around 11 different CD33r Siglecs (Siglec-3,-5,-6,-7,-8,-9,-10,-11,-14,-16,-17) while mice express only a few (Siglec-3,-E,-F,-G,-H) (11). Therefore, murine equivalents of only a few human Siglecs are known (Table 1).

\section{Siglec Affinities and Ligand Preferences}

Sialic acids are attached to glycans via $\alpha$-glycosidic linkages formed between its C2 with C3 $(\alpha 2,3)$ or C6 $(\alpha 2,6)$ positions of galactose or $\mathrm{C} 6$ position of $\mathrm{N}$-acetylgalactosamine. Polymers of SA are termed polysialic acid, where successive SA residues are primarily linked via $\alpha 2,8$ linkages, or by $\alpha 2,9$ linkages in a few cases (1). Different Siglecs have characteristic affinities towards specific sialoglycans based on the SA position, linkages, and surrounding sugars (Table 1). Siglec-9 recognizes SA linked to galactose via $\alpha 2,3 / \alpha 2,6$ linkages and other sialylated structures like sialyl Lewis-x (SLex), 6-sulfo-SLex $(22,23)$. However, Siglec8 prefers $\alpha 2,3$ linked SA attached to sulfated galactose, as seen in 6-sulfo-SLex (22). Ligand preferences of several Siglecs have been elucidated through glycan-binding arrays, molecular modeling studies, cell-based binding assays, etc. (22). Siglecs can bind sialoglycans present on the same cell (cis-interactions) or extracellular ligands present on neighboring cells or secretory glycoproteins (trans-interactions).

\section{Siglec Clustering Enhances Their Signaling Activity}

Siglec-sialoglycan binding is weak and transient (24). Interaction between Siglecs with multivalent ligands leads to Siglec clustering, which increases the strength of Siglec-ligand binding and initiates cellular signaling (22, 25-27). Multivalent ligands present several Siglec-binding sites. These may be extracellular ligands, antibodies, synthetic agonists interacting with Siglecs in trans, or cell surface sialoglycans, membranebound synthetic ligands binding in cis $(26,27)$. Siglec-clustering into nanodomains was revealed by high-resolution microscopy $(28,29)$. Siglec clustering into signaling domains by anti-Siglec antibodies is shown (Figure 1A).

\section{Function}

Siglecs are transmembrane proteins that generally interact with sialoglycans through the carbohydrate recognition domain present in their extracellular $\mathrm{N}$-terminal immunoglobulin-like folds (V-set domain). Several Siglecs contain immunoreceptor tyrosine-based inhibitory motifs (ITIMs) in their cytoplasmic domains while some are associated with adaptor proteins that contain immunoreceptor tyrosine-based activatory motifs (ITAMs) (7, 30). Signaling via ITIM leads to recruitment of Src homology region domain-containing phosphatase- 1 and 2 (SHP-1, SHP-2) which inhibits phosphorylation-based cellular signaling pathways $(14,31)$. Siglec-sialoglycan binding leads to modulation of cellular activity due to signaling via either ITIMs (inhibitory) or ITAMs (activatory) motifs (21).
Siglecs play several roles in normal physiology, including immunomodulation, phagocytosis of sialylated pathogens (mainly Siglec-1); regulation of B cell signaling and survival (CD22); maintenance of axon-myelin interactions, axon growth (Siglec-4); regulation of osteoclast differentiation (Siglec-15), etc (12-14, 16). Distribution, ligand preference, and functions of a few human Siglecs have been compiled in Table $\mathbf{1}$.

\section{Effect of ROS}

ROS has a direct detrimental effect on lipids, proteins, and DNA. However, low levels of ROS are required for maintaining metabolism, signal transduction, cell proliferation, apoptosis, and aging process (32). Oxidative stress due to excessive ROS generation is implicated in asthma, chronic obstructive pulmonary disease (COPD), diabetes, cardiovascular diseases, auto-immunity, and neurodegenerative diseases (5). Phagocytic cells express a multi-subunit NADPH-dependent phagocytic oxidase (Phox or NOX2), which produces ROS as a component of antimicrobial defense (33). Some components of NOX2 (p67 ${ }^{\text {phox }}, \mathrm{p} 47^{\text {phox }}, \mathrm{p} 40^{\text {phox }}$, a small G protein-rac1, rac2), are localized in the cytosol and some are membrane-associated (gp91 $\left.1^{\text {phox }}, \mathrm{p} 22^{\text {phox }}\right)$. The activity of membrane-associated gp91 ${ }^{\text {phox }}(34)$ is indispensable for NOX2 function, which uses $\mathrm{NADPH}$ as an electron donor to generate superoxide anion $\left(\mathrm{O}_{2}{ }^{*^{-}}\right)$by oxygen reduction (35-37).

\section{Siglec-Based Modulation of ROS Production}

Siglecs modulate ROS production through Siglec-sialoglycan binding, signaling through ITIMs or direct protein-protein interactions. Siglec- 9 was identified as a potential ligand for human amine oxidase type 3 (hAOC3) by phage peptide librarybased screening (38). Flow cytometry confirmed the binding between recombinant Chinese Hamster Ovary cells (CHO) expressing hAOC3 and CHO-expressing Siglec-9 (39). Surface plasmon resonance (SPR) based binding assays demonstrated that Siglec- $9 \mathrm{C}_{2}$ domain binds to the active site of hAOC3. Introduction of point mutations followed by SPR-based binding assays identified Arg284 and Arg290 in Siglec-9 to be critical for its binding with hAOC3. Such Siglec-binding with oxidase enzyme through protein-protein interactions enhances hAOC3 activity and generates hydrogen peroxide (39). Siglec-9 also binds to sialoglycoprotein hAOC3 through sialic acid-Siglec binding via its V domain (40).

Siglec-E knockout neutrophils infected with non-sialylated Escherichia coli strains (Gram-negative bacteria) produced significantly less amount of ROS than wild-type neutrophils, suggesting that Siglec-E promoted ROS generation (41). Silencing Siglec-E or Siglec-9 (the human equivalent of Siglec-E) also reduced ROS generation in neutrophils/THP-1 cells after E.coli infection (41). Immunoprecipitation revealed that endogenous Siglec-E associates with NOX2 subunits (gp $\left.91^{\text {phox }} / \mathrm{p} 47^{\text {phox }}\right)$ in E.coli-infected murine neutrophil and Tyr432 residue was found to be critical for Siglec-E-p47 $7^{\text {phox }}$ binding. Cells overexpressing Siglec-E with mutated Tyr432 also produced lower amounts of ROS following E.coli infection. 
A

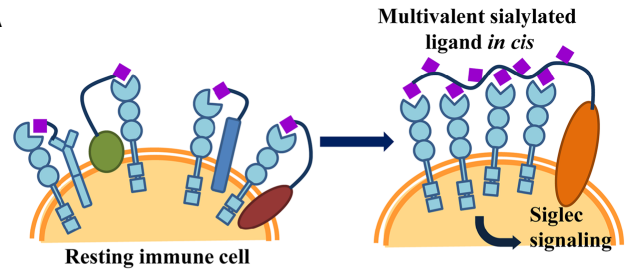

Multivalent sialylated Anti-Siglec antibodies

signaling

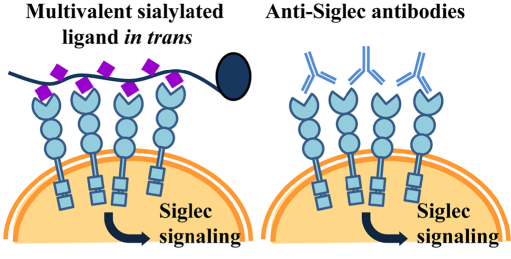

Clustering of Siglecs

B

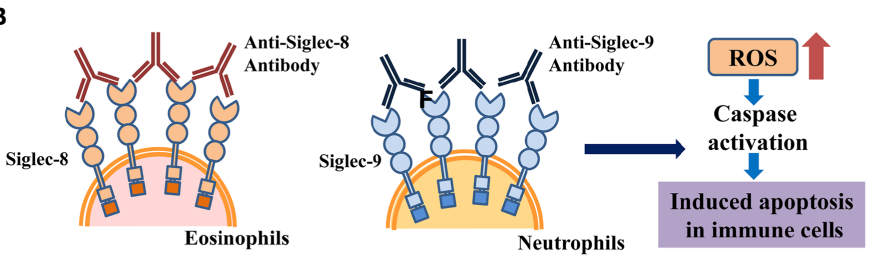

C

Uromodulin Increased Siglec-9 activation

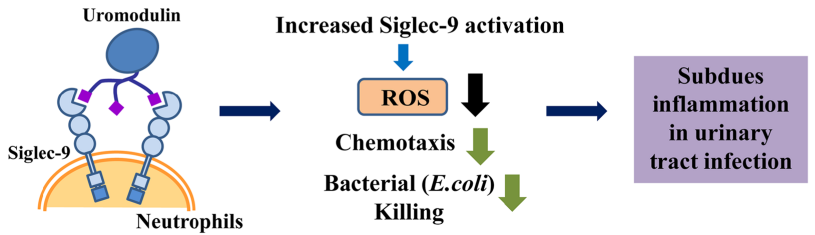

$\underset{\text { Sialic acids }}{\sim \text { Sialoglycan }}$

6 -protein of Group B Streptococcus (GBS)
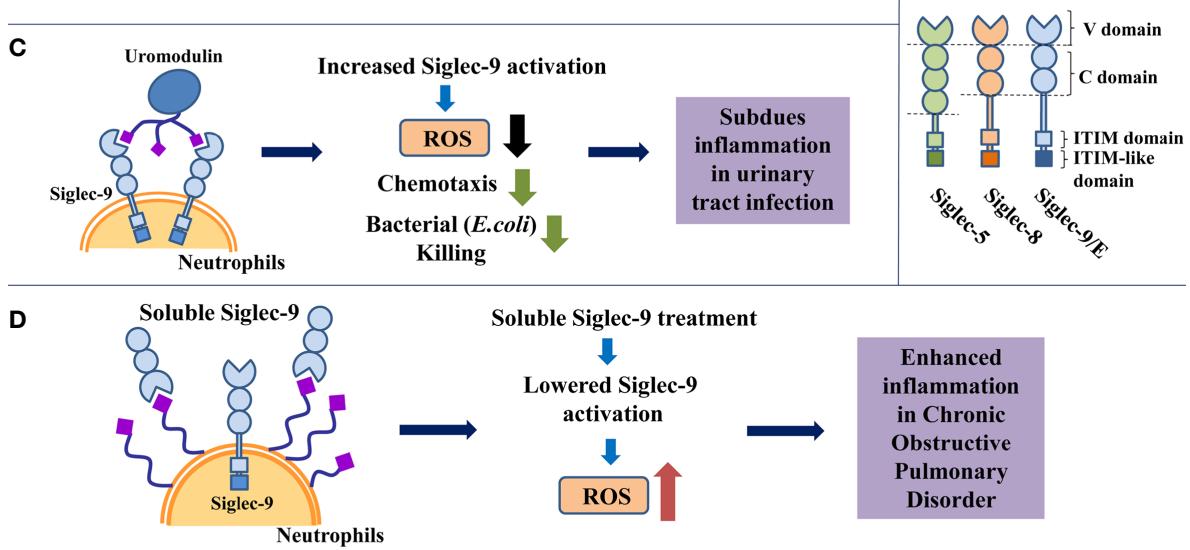

Soluble Siglec-9 treatment

$\downarrow$ Enhanced

Lowered Siglec-9

activation

inflammation

in Chronic

Obstructive

Pulmonary

Disorder

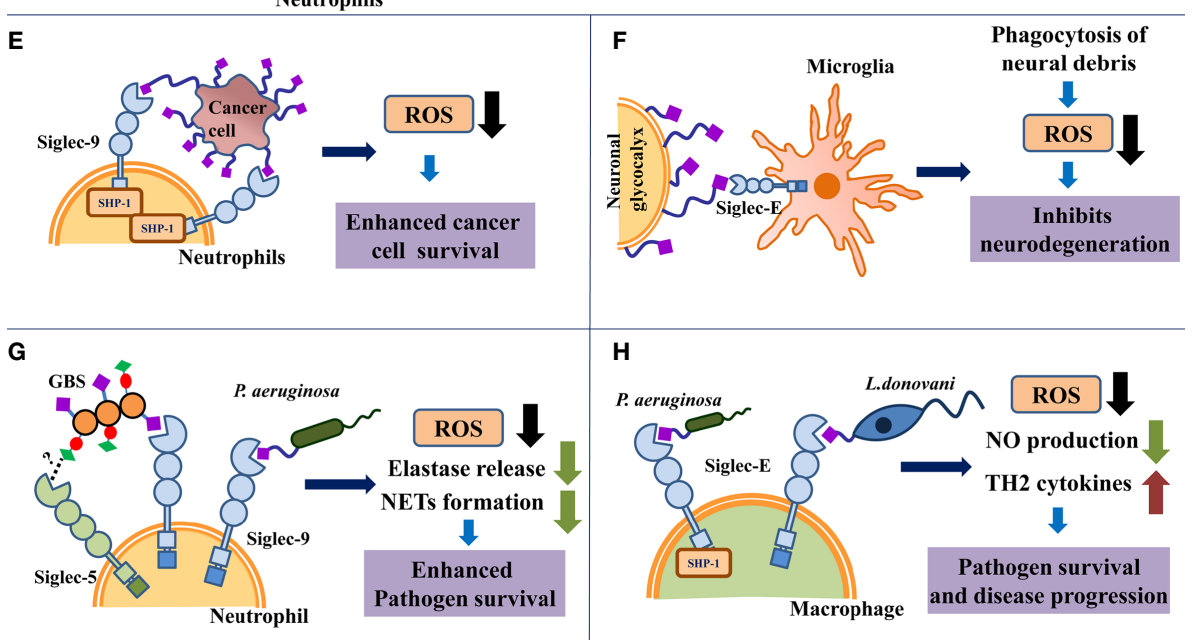

FIGURE 1 | Schematic representation of ROS modulation by Siglecs in immune cells under different pathological conditions. (A) Schematic representation of clustering of Siglecs on immune cell surface induced by interaction with various multivalent ligands. (B). Siglec-crosslinking using anti-Siglec antibodies promote ROS generation which triggers cellular apoptosis in resting neutrophils and eosinophils. (C) Uromodulin binding leads to enhanced Siglec-9 activation in neutrophils in vitro, inhibiting ROS production. This interaction possibly limits excessive inflammation during urinary tract infection. (D) Increased expression of soluble Siglec-9 (sSiglec-9) lowers Siglec-9-sialic acids engagement on neutrophils, leading to increased inflammation and ROS generation in chronic obstructive pulmonary disease (COPD). (E) Cancer cell sialoglycoproteins engage Siglecs on neutrophil via Siglec-sialoglycan interaction to reduce ROS production, which enhances cancer cell survival. (F) Siglec-E engagement with neural sialoglycoproteins suppresses ROS generation by microglial cells and prevents oxidative stress mediated neuro-degeneration. (G) Sialylated bacteria like Group B Streptococcus (GBS) and Pseudomonas aeruginosa interact with Siglec-9 present on neutrophil using sialic acids present on their surface. Additionally, non-sialylated GBS $\beta$-protein of GBS also binds with Siglec-5 present on neutrophil in a sialic acid-independent manner. Both Siglec-sialoglycan and Siglec-protein interactions encourage pathogen survival through subdued immune response. However, the exact binding site of protein mediated interaction between Siglec-5 and GBS $\beta$-protein, remains unknown. (H) Interaction of macrophage Siglec-E with sialylated pathogens like Pseudomonas aeruginosa and Leishmania donovani respectively subdues ROS production for disease progression. 
This confirmed that Tyr432 in the ITIM domain of Siglec-E is needed for its association with NOX2 subunit $\mathrm{p} 47^{\text {phox }}$ which promotes ROS production (41).

Interestingly, treatment of primary human neutrophils with a synthetic Siglec-9 agonist (pS9L) or anti-Siglec-9 antibody also leads to ROS generation which is inhibited upon SHP-1/2 inhibitor treatment. This indicates that Siglec- 9 engagement also promotes ROS generation through SHP-1/SHP-2 signaling (42).

\section{Siglec Engagement on Immune Cells Modulates ROS Generation Eosinophils}

Treating resting eosinophils with anti-Siglec- 8 monoclonal antibodies and secondary polyclonal antibodies leads to extensive crosslinking or clustering of Siglec-8 (Figure 1B, left panel). Siglec- 8 clustering was followed by ROS generation, reduction in mitochondrial membrane potential, and cleavage of caspases; culminating in cellular apoptosis $(43,44)$. Eosinophils incubated with pro-survival cytokine IL-5 show further increased cell death upon Siglec-8 cross-linking (45). These IL-5-activated eosinophils exhibit caspase-independent necrotic death, involving ROS generation and increased phosphorylation of MEK1, ERK1/2 (46). Treatment with ROS inhibitors confirmed that Siglec-crosslinking-mediated ROS production is essential for triggering eosinophil death in both resting and activated cells. Produced ROS was accumulated intracellularly in eosinophils (47).

In contrast, eosinophils stimulated with pro-survival cytokine IL-33 also exhibit enhanced cell death after Siglec-8 crosslinking, but without any significant increase in ROS generation (48). The lack of ROS production in IL-33-stimulated eosinophils remains to be explored.

Siglec-F shows a similar ligand binding profile like Siglec-8 and is also expressed on eosinophils. It is considered as a functionally convergent paralog of Siglec- 8 in mice $(49,50)$. Mice treated with anti-Siglec-F antibodies show induction of caspase-dependent eosinophil death independent of NADPH oxidase activity and ROS production (51).

Binding Siglec-8 with monoclonal antibodies or synthetic Siglec-8 ligands leads to increased expression of CD11b/CD18. CD11b/CD18 heterodimer belongs to the $\beta 2$-integrin subgroup, which increases the adhesiveness of IL-5-stimulated eosinophils. Such Siglec-8 engagement also leads to a time-dependent increase in ROS production, dependent on $\beta 2$-integrin expression (52). NADPH oxidase (NOX) enzyme was identified as the source of ROS (52). This indicates antibodies or ligand-based Siglec- 8 binding triggers ROS production through probable modulation of NOX activity.

\section{Neutrophils}

Neutrophils are the most abundant type of immune cells present in blood. Neutrophils perform immunosurveillance and respond to infiltrating microbes by phagocytosis, respiratory burst, degranulation, and formation of neutrophil extracellular traps (NETs) (53). Following successful clearance of microbes, responding neutrophils generally undergo apoptosis.
However, pro-survival cytokines or growth factors in the milieu often prevent such apoptosis, leading to chronic or acute inflammations.

Antibody-based cross-linking of Siglec-9 triggers apoptosis in normal resting neutrophils due to ROS generation and caspase cleavage (Figure 1B, right panel) (54). Interestingly, neutrophils isolated from patients suffering from inflammatory conditions like rheumatic arthritis, acute septic shock, etc. show enhanced expression of Siglec- 9 and increased death upon Siglec- 9 crosslinking. Neutrophils stimulated with GM-CSF, interferon- $\alpha$ or interferon- $\gamma($ IFN- $\alpha / \gamma)$ similarly exhibit increased cell death after Siglec- 9 cross-linking (47).

Such cytokine-stimulated neutrophils mainly showed nonapoptotic cell death upon Siglec-binding, characterized by cytoplasmic vacuolization, non-involvement of caspases, and only ROS generation. However, ROS generated by NADPH oxidase was identified to be crucial in both kinds of cell death (54).

Neutrophils express adhesion factors (like CD11b containing $\beta 2$-integrins) for attachment and migration from blood to other sites of injury or infection. They adhere to fibrinogen-coated plates via CD11b-fibrinogen binding, leading to integrintriggered ROS production (55). Additionally, Siglec-E binding with the sialoglycoprotein fibrinogen further increases ROS generation via NADPH-oxidase activity, which required fibrinogen-CD $11 \mathrm{~b}$ binding. In summary, Siglec- 8 in eosinophils (52) and Siglec-9/E in neutrophils (54, 55) modulate NOX activity for promoting ROS generation.

Additionally, Siglec-E-mediated $\beta 2$-integrin-dependent ROS generation prevented migration of neutrophils into the lungs of mice exposed to lipopolysaccharide, which was reversed upon inhibition of NOX activity (55).

\section{In Inflammation}

In normal physiology, Siglec-sialoglycan mediated signaling modulates immune cell activation, their response, ROS release, etc for preventing excessive inflammation and tissue injury (6).

\section{Urinary Tract Infections (UTI)}

Tamm-Horsfall protein (THP, uromodulin), is the most abundant protein in urine. It is a sialoglycoprotein that binds to Siglec-9/Siglec-E. Uromodulin treatment of neutrophils leads to reduced ROS generation, lowered chemotaxis, and reduced killing of uropathogenic Escherichia coli (56). During urinary tract infections, Uromodulin-Siglec-9 interaction possibly limits excessive neutrophil infiltration, reduces ROS generation, thereby modulating tissue inflammation (Figure 1C).

\section{Chronic Obstructive Pulmonary Disorder (COPD)}

Siglec-based regulation of ROS levels is observed in COPD patients. These patients exhibit elevated levels of the extracellular domain of Siglec-9 (soluble Siglec-9/sSiglec-9) in their plasma along with neutrophil hyperactivation, chemotaxis, and increased oxidative stress (57) (Figure 1D). Healthy neutrophils treated with sSiglec-9 in vitro show lower binding between neutrophil surface Siglec- 9 and sialoglycans and thereby reducing Siglec-9 activation. This leads to 
increased ROS production and chemotaxis by neutrophils (57). Expression of Siglec-9 was upregulated in peripheral blood and alveolar neutrophils in COPD patients, possibly to enhance Siglec-based inhibitory signaling in response to neutrophil hyperactivation (57).

\section{Sickle Cell Disease (SCD)}

Siglec-based regulation of neutrophil activation is also seen in sickle cell disease (SCD). Neutrophils cultured with healthy erythrocytes showed lowered activation and ROS generation. Healthy erythrocytes suppress neutrophil activation by engaging with neutrophil Siglec-9 via sialylated erythrocyte membrane proteins like glycophorin A (58). In SCD, erythrocytes rapidly age, losing membrane elasticity and undergoing changes in cell membrane composition and protein expression. SCD-erythrocytes contain more SA compared to healthy erythrocytes but show lowered binding with neutrophilSiglec-9 (59). Consequently, culturing healthy neutrophils with SCD erythrocytes leads to increased ROS release due to lowered Siglec-9 activation. Hyperactivation of neutrophils may lead to systemic inflammation, vaso-occlusion, etc. commonly observed in SCD.

\section{Cancer Progression}

Several types of cancers exhibit increased sialylation $(60,61)$. Neutrophils incubated with cancerous cells in vitro showed enhanced Siglec-9 engagement with cancer cell sialoglycoproteins, which increased SHP-1 recruitment. Consequently, ROS production and killing of tumor cell was inhibited (62) (Figure 1E). Mice lacking Siglec-E (murine equivalent of Siglec9) show increased neutrophil activity, immunosurveillance, and killing of injected tumor cells (62).

Surprisingly, established tumors grew quicker in mice lacking Siglec-E. Tumor-infiltrating macrophages in such mice showed an M2 type phenotype. Depending on environmental stimuli, macrophages are activated into M1 (pro-inflammatory, eliminates tumor cells) or M2 (promotes cell proliferation, wound repair) macrophages (63). Peritoneal macrophages from mice lacking Siglec-E showed upregulation of M2 polarization markers upon co-culture with cancerous cells. Therefore, depending on the stage of cancer progression, the absence of Siglec-E can enhance cancer cell killing (by neutrophil activity like ROS generation) or promote tumor growth (by inducing macrophage M2 polarization).

\section{Coronavirus Infections}

During the COVID-19 pandemic, coronavirus infections have been linked with uncontrolled inflammatory responses, hyperactivation of neutrophils, and NETs formation (64). Incubation with serum/plasma from COVID-19 patients triggered NETosis in neutrophils from healthy donors $(65,66)$. Siglec-9 engagement inhibits neutrophil activity and induces apoptosis. A Siglec-9 agonist, a glycopolypeptide bearing modified SA residues and lipid moieties (pS9L), induces Siglec9 clustering through cis interactions in macrophages after membrane insertion (28). Incubation of neutrophils with this agonist leads to ROS generation via SHP-1/2 and blocked
NETosis induced by COVID-19 plasma or TLR 7/9 agonists (42). This agonist may reduce inflammation by blocking NETosis and by triggering neutrophil apoptosis through ROS generation (54).

\section{Polysialic Acid-Based Nanoparticles}

Polysialic acids serve as multivalent ligands and activate Siglecs. Treatment of neutrophils with phorbol 12-myristate 13-acetate (PMA) induces NETosis and ROS generation. However, incubating neutrophils with aliphatic amine latex nanoparticles coupled to polysialic acids having $<9$ sialic acid residues, leads to a reduction in PMA-induced ROS levels and NETs formation (67). These polysialylated nanoparticles possibly function as ligands for neutrophil surface Siglec-5. Similarly, injection of polysialic acids having $\sim 20 \mathrm{SA}$ residues in transgenic mice prevented ROS generation by Siglec-11-expressing phagocytes. Age-related macular degeneration happens due to excessive ROS production, complement deposition which may be prevented by targeting siglec-11 via polysialic acids (68).

All these studies suggest that enhancing Siglec-sialoglycan engagement may be beneficial for controlling ROS levels in inflammatory disorders.

\section{Neurodegenerative Diseases}

Microglia are immune cells resident in the central nervous system, responsible for detecting invasive pathogens and maintaining tissue homeostasis by removing damaged, apoptotic or unnecessary synapses, neurons, and plaques. Microglial cells produce ROS following the phagocytosis of apoptotic bodies. However, oxidative stress in the central nervous system leads to neurodegenerative problems. SA content is highest in mammalian brains. Silencing Siglec-E expression in microglia confirmed that microglial Siglec-E binds to the sialylated neuronal glycocalyx to suppress ROS release following phagocytosis of neuronal debris (69). Here, Siglec-E regulates microglial ROS to prevent neurodegeneration (Figure 1F).

\section{Early Aging}

The lifespan of mammals is positively correlated with the number of CD33r Siglec genes (70). Siglecs are responsible for regulating ROS, particularly produced by activated phagocytes via NOX enzyme, during inflammatory responses. ROS possibly plays a role in early aging (71). Deletion of Siglec-E in mice resulted in shortened lifespan with increased generation of ROS and inhibition of ROS-detoxification systems (70). This correlation was confirmed across 26 species and held true for both activatory and inhibitory Siglecs (72).

Although a positive correlation exists between mammalian lifespan and siglecs, further research is needed to clearly understand how both the activatory and inhibitory siglecs are able to regulate the specific signaling pathways and receptors involved in neurodegenerative diseases.

\section{Down-Regulation of ROS in the Infection Process Through Siglec-Sialoglycan Interaction}

The capsular cell wall on Group B Streptococcus (GBS) is sialylated and associated with its virulence (73-77). These 
sialoglycans interact with Siglec-9 on human neutrophils for suppressing neutrophil oxidative burst and NETs formation, thus leading to enhanced bacterial survival (Figure 1G). A similar interaction was also reported with Siglec-E expressed on murine macrophages $(76,78)$.

Additionally, Group A Streptococcus expresses high molecular weight hyaluronan (HMW-HA). A similar sialylated molecule is also expressed on human neutrophils. Thus, Group A Streptococcus activates Siglec-9 on neutrophils through molecular mimicry to subdue ROS production, decrease NETs and apoptosis, for securing its existence inside the host $(76,79)$.

A few GBS strains express a non-sialylated $\beta$-protein docked on their cell wall that can efficiently engage Siglec-5 (Figure 1G). Such interaction was confirmed through the binding of SA-deficient mutant GBS with hSiglec-5-Fc. Trypsin treatment prevents this interaction by degrading the $\beta$-protein on the pathogen, indicating that binding was protein-mediated and independent of SA (75). It remains to be explored how non-sialylated proteins bind to Siglecs, as the binding site and the mechanism of such binding is still unknown. This association activated the inhibitory signaling through the engagement of SHP1/2. Thus, suppressed host immune responses like oxidative burst, repressed phagocytic activity, and extracellular traps in the leucocytes, thereby favoring pathogen persistence (75).

Likewise, we had reported the presence of SA in Pseudomonas aeruginosa (PA) and identified a few sialoglycoproteins by mass spectrometry (80). These sialoglycoproteins interact with several human Siglecs via SA to enhance their pathogenicity (81-83). Interaction of $P A$-SA with inhibitory Siglec-9 on neutrophils reduced ROS production, elastase release, and decreased NETs formation, which altogether suppressed the activation of these immune cells (Figure 1G). Moreover, the association of the bacterial SA with murine Siglec-E on macrophages exhibited enhanced phagocytosis but reduced oxidative burst (Figure 1H) (83).

Our group also had demonstrated that Leishmania donovani, the causative agent of Indian visceral leishmaniasis displays different derivatives of sialic acid on its cell surface (84). Subsequently, it was also shown that various strains of Leishmania species causing different forms of the disease exhibited a differential distribution of SA on their surface. Binding studies of sialylated virulent L.donovani strain with soluble siglec-Fc chimeras displayed its high interaction only with Siglec-1 and Siglec-5. Furthermore, we also reported that these parasites interact with Siglec-E on murine macrophages to subvert the host immune response $(85,86)$. ROS production and other macrophage effector functions were upregulated by silencing Siglec-E, which ultimately diminished the parasite survival inside the host (83). This indicates that Siglec-E suppresses ROS production in parasite-infected macrophages (Figure 1H).

All this information supports the well-established immuneinhibitory role of Siglecs in the promotion of pathogen survival and disease progression. Thus, Siglecs at the host-pathogen interface can play a very important role in modulating the immune response through regulating ROS production in the immune cells.

\section{DISCUSSION}

The crucial role of Siglec-sialoglycan interactions in regulating immune and inflammatory responses is increasingly becoming relevant. Siglecs are being recognized as potential therapeutic targets in various inflammatory disorders and cancer (87, 88). Strategies to target activatory or inhibitory Siglecs for regulating immune response involve modulation of overall sialylation, use of antibodies, or sialic acid mimetics (89-93).

Several studies demonstrate that Siglec-sialoglycan interactions additionally promote as well as inhibit ROS generation to control diverse cellular functions ranging from apoptosis to maintenance of cellular life-spans. Such interactions may be beneficial for modulating oxidative stress as a part of anti-tumor, anti-inflammatory therapy. Siglecs may induce immune-tolerance or suppress inflammation by depleting ROS-producing cells or inhibiting ROS release. A few Siglec agonists which enhanced Siglec-based suppression of ROS production in inflammatory disorders have already been demonstrated (28, 42, 67-69).

However, the consequence of Siglec-sialoglycan interaction may vary depending upon the location of Siglec and the presence of its cognate ligand in the surroundings. Also, some of the Siglecs have redundant roles. More comprehensive studies are required to define the outcome of such signaling events before siglecs may be used as therapeutic agents. In this respect, the role of Siglec-sialoglycan interactions and protein-protein interactions in Siglec-based ROS regulation needs to be explored in detail.

\section{AUTHOR CONTRIBUTIONS}

All the authors enlisted have made substantial, direct, and intellectual contribution to the work and approved it in its final form.

\section{ACKNOWLEDGMENTS}

$\mathrm{JK}$ and $\mathrm{KM}$ are supported from Science and Engineering Research Board (SERB)- funded project. CM is grateful for the financial support from Distinguished Fellow (SERB), Sir J.C. Bose National Fellowship, Department of Science and Technology (DST). The work is partially supported by CSIRHCP010. We sincerely acknowledge the Director, CSIR-Indian Institute of Chemical Biology, Kolkata, India for providing all institute facilities. 


\section{REFERENCES}

1. Schauer R, Kamerling JP. Exploration of the Sialic Acid World. Adv Carbohydr Chem Biochem (2018) 75:1-213. doi: 10.1016/bs.accb.2018.09.001

2. Crocker PR, Clark EA, Filbin M, Gordon S, Jones Y, Kehrl JH, et al. Siglecs: A Family of Sialic-Acid Binding Lectins. Glycobiol (1998) 8:v. doi: 10.1093/ oxfordjournals.glycob.a018832

3. Lübbers J, Rodríguez E, van Kooyk Y. Modulation of Immune Tolerance via Siglec-Sialic Acid Interactions. Front Immunol (2018) 9:2807. doi: 10.3389/ fimmu.2018.02807

4. Gianchecchi E, Arena A, Fierabracci A. Sialic Acid-Siglec Axis in Human Immune Regulation, Involvement in Autoimmunity and Cancer and Potential Therapeutic Treatments. Int J Mol Sci (2021) 22:5774. doi: 10.3390/ijms22115774

5. Selemidis S. Targeting Reactive Oxygen Species for Respiratory Infection: Fact Or Fancy? Respirol (2019) 24:15-6. doi: 10.1111/resp.13417

6. Novaes RD, Teixeira AL, de Miranda AS. Oxidative Stress in Microbial Diseases: Pathogen, Host, and Therapeutics. Oxid Med Cell Longev (2019) 2019:8159562. doi: 10.1155/2019/8159562

7. von Gunten S, Bochner BS. Basic and Clinical Immunology of Siglecs. Ann N Y Acad Sci (2008) 1143:61-82. doi: 10.1196/annals.1443.011

8. Nguyen DH, Hurtado-Ziola N, Gagneux P, Varki A. Loss of Siglec Expression on T Lymphocytes During Human Evolution. Proc Natl Acad Sci USA (2006) 103:7765-70. doi: 10.1073/pnas.0510484103

9. Läubli H, Varki A. Sialic Acid-Binding Immunoglobulin-Like Lectins (Siglecs) Detect Self-Associated Molecular Patterns to Regulate Immune Responses. Cell Mol Life Sci (2020) 77:593-605. doi: 10.1007/s00018-01903288- $\mathrm{x}$

10. Smith BAH, Bertozzi CR. The Clinical Impact of Glycobiology: Targeting Selectins, Siglecs and Mammalian Glycans. Nat Rev Drug Discovery (2021) 20:217-43. doi: 10.1038/s41573-020-00093-1

11. Lin $\mathrm{CH}$, Yeh YC, Yang KD. Functions and Therapeutic Targets of SiglecMediated Infections, Inflammations and Cancers. J Formos Med Assoc (2021) 120:5-24. doi: 10.1016/j.jfma.2019.10.019

12. Varki A, Angata T. Siglecs-the Major Subfamily of I-Type Lectins. Glycobiol (2006) 16:1R-27R. doi: 10.1093/glycob/cwj008

13. Duan S, Paulson JC. Siglecs as Immune Cell Checkpoints in Disease. Annu Rev Immunol (2020) 38:365-95. doi: 10.1146/annurev-immunol-102419-035900

14. Crocker PR, Paulson JC, Varki A. Siglecs and Their Roles in the Immune System. Nat Rev Immunol (2007) 7:255-66. doi: 10.1038/nri2056

15. Perez-Zsolt D, Martinez-Picado J, Izquierdo-Useros N. When Dendritic Cells Go Viral: The Role of Siglec-1 in Host Defense and Dissemination of Enveloped Viruses. Viruses (2019) 12:8. doi: 10.3390/v12010008

16. Macauley MS, Crocker PR, Paulson JC. Siglec-Mediated Regulation of Immune Cell Function in Disease. Nat Rev Immunol (2014) 14:653-66. doi: 10.1038/nri3737

17. Yokoi H, Myers A, Matsumoto K, Crocker PR, Saito H, Bochner BS. Alteration and Acquisition of Siglecs During In Vitro Maturation of CD34+ Progenitors Into Human Mast Cells. Allergy (2006) 61:769-76. doi: 10.1111/ j.1398-9995.2006.01133.x

18. Toba K, Hanawa H, Fuse I, Sakaue M, Watanabe K, Uesugi Y, et al. Difference in CD22 Molecules in Human B Cells and Basophils. Exp Hematol (2002) 30:205-11. doi: 10.1016/s0301-472x(01)00791-3

19. Wen T, Mingler MK, Blanchard C, Wahl B, Pabst O, Rothenberg ME. The Pan-B Cell Marker CD22 Is Expressed on Gastrointestinal Eosinophils and Negatively Regulates Tissue Eosinophilia. J Immunol (2012) 188:1075-82. doi: 10.4049/jimmunol.1102222

20. Clark EA, Giltiay NV. CD22: A Regulator of Innate and Adaptive B Cell Responses and Autoimmunity. Front Immunol (2018) 9:2235. doi: 10.3389/ fimmu.2018.02235

21. Bornhöfft KF, Goldammer T, Rebl A, Galuska SP. Siglecs: A Journey Through the Evolution of Sialic Acid-Binding Immunoglobulin-Type Lectins. Dev Comp Immunol (2018) 86:219-31. doi: 10.1016/j.dci.2018.05.008

22. Gonzalez-Gil A, Schnaar RL. Siglec Ligands. Cells (2021) 10:1260. doi: 10.3390/cells10051260

23. Zhang JQ, Nicoll G, Jones C, Crocker PR. Siglec-9, A Novel Sialic Acid Binding Member of the Immunoglobulin Superfamily Expressed Broadly on
Human Blood Leukocytes. J Biol Chem (2000) 275:22121-6. doi: 10.1074/ jbc.M002788200

24. Yamakawa N, Yasuda Y, Yoshimura A, Goshima A, Crocker PR, Vergoten G, et al. Discovery of a New Sialic Acid Binding Region That Regulates Siglec-7. Sci Rep (2020) 10:8647. doi: 10.1038/s41598-020-64887-4

25. Avril T, Attrill H, Zhang J, Raper A, Crocker PR. Negative Regulation of Leucocyte Functions by CD33-Related Siglecs. Biochem Soc Trans (2006) 34:1024-7. doi: 10.1042/BST0341024

26. Collins BE, Paulson JC. Cell Surface Biology Mediated by Low Affinity Multivalent Protein-Glycan Interactions. Curr Opin Chem Biol (2004) 8:617-25. doi: 10.1016/j.cbpa.2004.10.004

27. O'Reilly MK, Paulson JC. Multivalent Ligands for Siglecs. Methods Enzymol (2010) 478:343-63. doi: 10.1016/S0076-6879(10)78017-4

28. Delaveris CS, Chiu SH, Riley NM, Bertozzi CR. Modulation of Immune Cell Reactivity With Cis-Binding Siglec Agonists. Proc Natl Acad Sci USA (2021) 118:e2012408118. doi: 10.1073/pnas.2012408118

29. Gasparrini F, Feest C, Bruckbauer A, Mattila PK, Müller J, Nitschke L, et al. Nanoscale Organization and Dynamics of the Siglec CD22 Cooperate With the Cytoskeleton in Restraining BCR Signalling. EMBO J (2016) 35:258-80. doi: 10.15252/embj.201593027

30. Angata T. Siglecs That Associate With DAP12. Adv Exp Med Biol (2020) 1204:215-30. doi: 10.1007/978-981-15-1580-4_9

31. Cao H, Crocker PR. Evolution of CD33-Related Siglecs: Regulating Host Immune Functions and Escaping Pathogen Exploitation? Immunol (2011) 132:18-26. doi: 10.1111/j.1365-2567

32. Nogueira V, Hay N. Molecular Pathways: Reactive Oxygen Species Homeostasis in Cancer Cells and Implications for Cancer Therapy. Clin Cancer Res (2013) 19:4309-14. doi: 10.1158/1078-0432.CCR-12-1424

33. Segal AW. The Function of the NADPH Oxidase of Phagocytes and Its Relationship to Other Noxs in Plants, Invertebrates, And Mammals. Int $J$ Biochem Cell Biol (2008) 40:604-18. doi: 10.1016/j.biocel.2007.10.003

34. Cachat J, Deffert C, Hugues S, Krause KH. Phagocyte NADPH Oxidase and Specific Immunity. Clin Sci (Lond) (2015) 128:635-48. doi: 10.1042/ CS20140635

35. Roma EH, Macedo JP, Goes GR, Gonçalves JL, Castro WD, Cisalpino D, et al. Impact of Reactive Oxygen Species (ROS) on the Control of Parasite Loads and Inflammation in Leishmania Amazonensis Infection. Parasit Vectors (2016) 9:193. doi: 10.1186/s13071-016-1472-y

36. Babior BM. NADPH Oxidase: An Update. Blood (1999) 93:1464-76. doi: 10.1182/blood.V93.5.1464

37. Shatwell KP, Segal AW. NADPH Oxidase. Int J Biochem Cell Biol (1996) 28:1191-5. doi: 10.1016/s1357-2725(96)00084-2

38. Aalto K, Autio A, Kiss EA, Elima K, Nymalm Y, Veres TZ, et al. Siglec-9 Is a Novel Leukocyte Ligand for Vascular Adhesion Protein-1 and Can be Used in PET Imaging of Inflammation and Cancer. Blood (2011) 118:3725-33. doi: 10.1182/blood-2010-09-311076

39. Elovaara H, Parkash V, Fair-Mäkelä R, Salo-Ahen OM, Guédez G, BligtLindén E, et al. Multivalent Interactions of Human Primary Amine Oxidase With the $\mathrm{V}$ and $\mathrm{C}_{2}$ Domains of Sialic Acid-Binding Immunoglobulin-Like Lectin-9 Regulate Its Binding and Amine Oxidase Activity. PloS One (2016) 11:e0166935. doi: 10.1371/journal.pone.0166935

40. Lopes de Carvalho L, Elovaara H, de Ruyck J, Vergoten G, Jalkanen S, Guédez G, et al. Mapping the Interaction Site and Effect of the Siglec-9 Inflammatory Biomarker On Human Primary Amine Oxidase. Sci Rep (2018) 1(8):2086. doi: 10.1038/s41598-018-20618-4

41. Wu Y, Yang D, Liu R, Wang L, Chen GY. Selective Response to Bacterial Infection by Regulating Siglec-E Expression. iScience (2020) 23:101473. doi: $10.1016 /$ j.isci.2020.101473

42. Delaveris CS, Wilk AJ, Riley NM, Stark JC, Yang SS, Rogers AJ, et al. Synthetic Siglec-9 Agonists Inhibit Neutrophil Activation Associated With COVID-19. ACS Cent Sci (2021) 7:650-7. doi: 10.1021/acscentsci.0c01669

43. Nutku E, Aizawa H, Hudson SA, Bochner BS. Ligation of Siglec-8: A Selective Mechanism For Induction of Human Eosinophil Apoptosis. Blood (2003) 101:5014-20. doi: 10.1182/blood-2002-10-3058

44. Nutku E, Hudson SA, Bochner BS. Mechanism of Siglec-8-Induced Human Eosinophil Apoptosis: Role of Caspases and Mitochondrial Injury. Biochem Biophys Res Commun (2005) 336:918-24. doi: 10.1016/j.bbrc.2005.08.202 
45. Nutku-Bilir E, Hudson SA, Bochner BS. Interleukin-5 Priming of Human Eosinophils Alters Siglec-8 Mediated Apoptosis Pathways. Am J Respir Cell Mol Biol (2008) 1:121-4. doi: 10.1165/rcmb.2007-0154OC

46. Kano G, Almanan M, Bochner BS, Zimmermann N. Mechanism of Siglec-8Mediated Cell Death in IL-5-Activated Eosinophils: Role for Reactive Oxygen Species-Enhanced MEK/ERK Activation. J Allergy Clin Immunol (2013) 132:437-45. doi: 10.1016/j.jaci.2013.03.024

47. Kano G, Bochner BS, Zimmermann N. Regulation of Siglec-8-Induced Intracellular Reactive Oxygen Species Production and Eosinophil Cell Death by Src Family Kinases. Immunobiol (2017) 222:343-9. doi: 10.1016/ j.imbio.2016.09.006

48. Na HJ, Hudson SA, Bochner BS. IL-33 Enhances Siglec-8 Mediated Apoptosis of Human Eosinophils. Cytokine (2012) 57:169-74. doi: 10.1016/ j.cyto.2011.10.007

49. Farid SSH, Mirshafiey A, Razavi A. Siglec-8 and Siglec-F, the New Therapeutic Targets in Asthma. Immunopharmacol Immunotoxicol (2012) 34:721-6. doi: 10.3109/08923973.2011.589453

50. Tateno H, Crocker PR, Paulson JC. Mouse Siglec-F and Human Siglec-8 are Functionally Convergent Paralogs That Are Selectively Expressed on Eosinophils and Recognize 6'-Sulfo-Sialyl Lewis X as a Preferred Glycan Ligand. Glycobiology (2005) 15:1125-35. doi: 10.1093/glycob/cwi097

51. Mao H, Kano G, Hudson SA, Brummet M, Zimmermann N, Zhu Z, et al. Mechanisms of Siglec-F-Induced Eosinophil Apoptosis: A Role for Caspases But Not for SHP-1, Src Kinases, NADPH Oxidase or Reactive Oxygen. PloS One (2013) 8:e68143. doi: 10.1371/journal.pone.0068143

52. Carroll DJ, O'Sullivan JA, Nix DB, Cao Y, Tiemeyer M, Bochner BS. Sialic Acid-Binding Immunoglobulin-Like Lectin 8 (Siglec-8) Is an Activating Receptor Mediating $\beta 2$-Integrin-Dependent Function in Human Eosinophils. J Allergy Clin Immunol (2018) 141:2196-207. doi: 10.1016/ j.jaci.2017.08.013

53. Kolaczkowska E, Kubes P. Neutrophil Recruitment and Function in Health and Inflammation. Nat Rev Immunol (2013) 13:159-75. doi: 10.1038/nri3399

54. von Gunten S, Yousefi S, Seitz M, Jakob SM, Schaffner T, Seger R, et al. Siglec9 Transduces Apoptotic and Nonapoptotic Death Signals Into Neutrophils Depending on the Proinflammatory Cytokine Environment. Blood (2005) 106:1423-31. doi: 10.1182/blood-2004-10-4112

55. McMillan SJ, Sharma RS, Richards HE, Hegde V, Crocker PR. Siglec-E Promotes B2-Integrin-Dependent NADPH Oxidase Activation to Suppress Neutrophil Recruitment to the Lung. J Biol Chem (2014) 289:20370-6. doi: 10.1074/jbc.M114.574624

56. Patras KA, Coady A, Olson J, Ali SR, RamachandraRao SP, Kumar S, et al. Tamm-Horsfall Glycoprotein Engages Human Siglec-9 to Modulate Neutrophil Activation in the Urinary Tract. Immunol Cell Biol (2017) 95:960-5. doi: 10.1038/icb.2017.63

57. Zeng Z, Li M, Wang M, Wu X, Li Q, Ning Q, et al. Increased Expression of Siglec-9 in Chronic Obstructive Pulmonary Disease. Sci Rep (2017) 7:10116. doi: 10.1038/s41598-017-09120-5

58. Lizcano A, Secundino I, Döhrmann S, Corriden R, Rohena C, Diaz S, et al. A. Erythrocyte Sialoglycoproteins Engage Siglec-9 on Neutrophils to Suppress Activation. Blood (2017) 129:3100-10. doi: 10.1182/blood-2016-11-751636

59. Kiser ZM, Lizcano A, Nguyen J, Becker GL, Belcher JD, Varki AP, et al. Decreased Erythrocyte Binding of Siglec-9 Increases Neutrophil Activation in Sickle Cell Disease. Blood Cells Mol Dis (2020) 81:102399. doi: 10.1016/ j.bcmd.2019.102399

60. Mandal C, Chatterjee M, Sinha D. Investigation of 9-O-Acetylated Sialoglycoconjugates in Childhood Acute Lymphoblastic Leukaemia. $\mathrm{Br} J$ Haematol (2000) 110:801-12. doi: 10.1046/j.1365-2141.2000.02105.x

61. Sinha D, Mandal C, Bhattacharya DK. Identification of 9-O Acetyl Sialoglycoconjugates (9-OAcSGs) as Biomarkers in Childhood Acute Lymphoblastic Leukemia Using a Lectin, AchatininH, as a Probe. Leukemia (1999) 13:119-25. doi: 10.1038/sj.leu.2401239

62. Läubli H, Pearce OM, Schwarz F, Siddiqui SS, Deng L, Stanczak MA, et al. Engagement of Myelomonocytic Siglecs by Tumor-Associated Ligands Modulates the Innate Immune Response to Cancer. Proc Natl Acad Sci USA (2014) 111:14211-6. doi: 10.1073/pnas.1409580111

63. Sica A, Larghi P, Mancino A, Rubino L, Porta C, Totaro MG, et al. Macrophage Polarization in Tumour Progression. Semin Cancer Biol (2008) 18:349-55. doi: 10.1016/j.semcancer.2008.03.004
64. Thierry AR, Roch B. Neutrophil Extracellular Traps and By-Products Play a Key Role in COVID-19: Pathogenesis, Risk Factors, and Therapy. J Clin Med (2020) 9:2942. doi: 10.3390/jcm9092942

65. Zuo Y, Yalavarthi S, Shi H, Gockman K, Zuo M, Madison JA, et al. Neutrophil Extracellular Traps in COVID-19. JCI Insight (2020) 5(11):e138999. doi: 10.1172/jci.insight.138999

66. Middleton EA, He XY, Denorme F, Campbell RA, Ng D, Salvatore SP, et al. Neutrophil Extracellular Traps Contribute to Immunothrombosis in COVID19 Acute Respiratory Distress Syndrome. Blood (2020) 136(10):1169-79. doi: 10.1182/blood.2020007008

67. Bornhöfft KF, Viergutz T, Kühnle A, Galuska SP. Nanoparticles Equipped With $\alpha 2,8$-Linked Sialic Acid Chains Inhibit the Release of Neutrophil Extracellular Traps. Nanomaterials (Basel) (2019) 9:610. doi: 10.3390/ nano9040610

68. Karlstetter M, Kopatz J, Aslanidis A, Shahraz A, Caramoy A, Linnartz-Gerlach B, et al. Polysialic Acid Blocks Mononuclear Phagocyte Reactivity, Inhibits Complement Activation, and Protects From Vascular Damage In The Retina. EMBO Mol Med (2017) 9:154-66. doi: 10.15252/emmm.201606627

69. Claude J, Linnartz-Gerlach B, Kudin AP, Kunz WS, Neumann H. Microglial CD33-Related Siglec-E Inhibits Neurotoxicity by Preventing the Phagocytosis-Associated Oxidative Burst. J Neurosci (2013) 33:18270-6. doi: 10.1523/JNEUROSCI.2211-13.2013

70. Schwarz F, Pearce OM, Wang X, Samraj AN, Läubli H, Garcia JO, et al. Siglec Receptors Impact Mammalian Lifespan by Modulating Oxidative Stress. Elife (2015) 4:e06184. doi: 10.7554/eLife.06184

71. Liguori I, Russo G, Curcio F, Bulli G, Aran L, Della-Morte D, et al. Oxidative Stress, Aging, and Diseases. Clin Interv Aging (2018) 13:757-72. doi: 10.2147/ CIA.S158513

72. Khan N, Kim SK, Gagneux P, Dugan LL, Varki A. Maximum Reproductive Lifespan Correlates With CD33rSIGLEC Gene Number: Implications for NADPH Oxidase-Derived Reactive Oxygen Species in Aging. FASEB J (2020) 34:1928-38. doi: 10.1096/fj.201902116R

73. Uchiyama S, Sun J, Fukahori K, Ando N, Wu M, Schwarz F, et al. Dual Actions of Group B Streptococcus Capsular Sialic Acid Provide Resistance to Platelet-Mediated Antimicrobial Killing. Proc Natl Acad Sci USA (2019) 116:7465-70. doi: 10.1073/pnas.1815572116.

74. Carlin AF, Lewis AL, Varki A, Nizet V. Group B Streptococcal Capsular Sialic Acids Interact With Siglecs (Immunoglobulin-Like Lectins) on Human Leukocytes. J Bacteriol (2007) 189:1231-7. doi: 10.1128/JB.01155-06

75. Carlin AF, Chang YC, Areschoug T, Lindahl G, Hurtado-Ziola N, King CC, et al. B Streptococcus Suppression of Phagocyte Functions by ProteinMediated Engagement of Human Siglec-5. J Exp Med (2009) 206:1691-9. doi: 10.1084/jem.20090691

76. Carlin AF, Uchiyama S, Chang YC, Lewis AL, Nizet V, Varki A. Molecular Mimicry of Host Sialylated Glycans Allows a Bacterial Pathogen to Engage Neutrophil Siglec-9 and Dampen the Innate Immune Response. Blood (2009) 113:3333-6. doi: 10.1182/blood-2008-11-187302

77. Wessels MR, Rubens CE, Benedí VJ, Kasper DL. Definition of a Bacterial Virulence Factor: Sialylation of the Group B Streptococcal Capsule. Proc Natl Acad Sci USA (1989) 86:8983-7. doi: 10.1073/pnas.86.22.8983

78. Chang YC, Olson J, Beasley FC, Tung C, Zhang J, Crocker PR, et al. Group B Streptococcus Engages An Inhibitory Siglec Through Sialic Acid Mimicry to Blunt Innate Immune and Inflammatory Responses In Vivo. PloS Pathog (2014) 10:e1003846. doi: 10.1371/journal.ppat.1003846

79. Secundino I, Lizcano A, Roupé KM, Wang X, Cole JN, Olson J, et al. Host and Pathogen Hyaluronan Signal Through Human Siglec-9 to Suppress Neutrophil Activation. J Mol Med (Berl) (2016) 94:219-33. doi: 10.1007/ s00109-015-1341-8

80. Khatua B, Van Vleet J, Choudhury BP, Chaudhry R, Mandal C. Sialylation of Outer Membrane Porin Protein D: A Mechanistic Basis of Antibiotic Uptake in Pseudomonas Aeruginosa. Mol Cell Proteomics (2014) 13:1412-28. doi: 10.1074/mcp.M113.030999

81. Khatua B, Ghoshal A, Bhattacharya K, Mandal C, Saha B, Crocker PR, et al. Sialic Acids Acquired by Pseudomonas Aeruginosa Are Involved in Reduced Complement Deposition and Siglec Mediated Host-Cell Recognition. FEBS Lett (2010) 584:555-61. doi: 10.1016/j.febslet.2009.11.087

82. Khatua B, Bhattacharya K, Mandal C. Sialoglycoproteins Adsorbed by Pseudomonas Aeruginosa Facilitate Their Survival by Impeding Neutrophil 
Extracellular Trap Through Siglec-9. J Leukoc Biol (2012) 91:641-55. doi: $10.1189 / \mathrm{jlb} .0511260$.

83. Mukherjee K, Khatua B, Mandal C. Sialic Acid-Siglec-E Interactions During Pseudomonas Aeruginosa Infection of Macrophages Interferes With Phagosome Maturation by Altering Intracellular Calcium Concentrations. Front Immunol (2020) 11:332. doi: 10.3389/fimmu.2020.00332

84. Chatterjee M, Chava AK, Kohla G, Pal S, Merling A, Hinderlich S, et al. Identification and Characterization of Adsorbed Serum Sialoglycans on Leishmania Donovani Promastigotes. Glycobiol (2003) 13:351-61. doi: $10.1093 /$ glycob/cwg027

85. Roy S, Mandal C. Leishmania Donovani Utilize Sialic Acids for Binding and Phagocytosis in the Macrophages Through Selective Utilization of Siglecs and Impair the Innate Immune Arm. PloS Negl Trop Dis (2016) 10:e0004904. doi: 10.1371/journal.pntd.0004904

86. Karmakar J, Mandal C. Interplay Between Sialic Acids, Siglec-E, and Neul Regulates MyD88- and TRIF-Dependent Pathways for TLR4-Activation During Leishmania Donovani Infection. Front Immunol (2021) 12:626110. doi: $10.3389 /$ fimmu.2021.626110

87. Lenza MP, Atxabal U, Oyenarte I, Jiménez-Barbero J, Ereño-Orbea J. Current Status on Therapeutic Molecules Targeting Siglec Receptors. Cells (2020) 9:2691. doi: 10.3390/cells9122691

88. O'Reilly MK, Paulson JC. Siglecs as Targets for Therapy in Immune-CellMediated Disease. Trends Pharmacol Sci (2009) 30:240-8. doi: 10.1016/ j.tips.2009.02.005

89. Stanczak MA, Siddiqui SS, Trefny MP, Thommen DS, Boligan KF, von Gunten S, et al. Self-Associated Molecular Patterns Mediate Cancer Immune Evasion by Engaging Siglecs on T Cells. J Clin Invest (2018) 128 (11):4912-23. doi: 10.1172/JCI120612

90. Perdicchio M, Cornelissen LA, Streng-Ouwehand I, Engels S, Verstege MI, Boon L, et al. Tumor Sialylation Impedes T Cell Mediated Anti-Tumor
Responses While Promoting Tumor Associated-Regulatory T Cells. Oncotarget (2016) 7(8):8771-82. doi: 10.18632/oncotarget.6822

91. Gray MA, Stanczak MA, Mantuano NR, Xiao H, Pijnenborg JFA, Malaker SA, et al. Targeted Glycan Degradation Potentiates the Anticancer Immune Response In Vivo. Nat Chem Biol (2020) 16(12):1376-84. doi: 10.1038/s41589-020-0622-x

92. Schweizer A, Wohner M, Prescher H, Brossmer R, Nitschke L. Targeting of CD22Positive B-Cell Lymphoma Cells by Synthetic Divalent Sialic Acid Analogues. Eur J Immunol (2012) 42(10):2792-802. doi: 10.1002/eji.201242574

93. Prescher H, Schweizer A, Kuhfeldt E, Nitschke L, Brossmer R. Discovery of Multifold Modified Sialosides as Human CD22/ Siglec-2 Ligands With Nanomolar Activity on B-Cells. ACS Chem Biol (2014) 9(7):1444-50. doi: $10.1021 / \mathrm{cb} 400952 \mathrm{v}$

Conflict of Interest: The authors declare that the research was conducted in the absence of any commercial or financial relationships that could be construed as a potential conflict of interest.

Publisher's Note: All claims expressed in this article are solely those of the authors and do not necessarily represent those of their affiliated organizations, or those of the publisher, the editors and the reviewers. Any product that may be evaluated in this article, or claim that may be made by its manufacturer, is not guaranteed or endorsed by the publisher.

Copyright $\odot 2021$ Karmakar, Mukherjee and Mandal. This is an open-access article distributed under the terms of the Creative Commons Attribution License (CC BY). The use, distribution or reproduction in other forums is permitted, provided the original author(s) and the copyright owner(s) are credited and that the original publication in this journal is cited, in accordance with accepted academic practice. No use, distribution or reproduction is permitted which does not comply with these terms. 